\title{
The Growth Response of Cellulolytic Acetate-utilizing and Acetate- producing Butyrivibrios to Volatile Fatty Acids and Other Nutrients
}

\author{
By C. ROCHÉ, HANTIE ALBERTYN, N. O. VAN GYLSWYK \\ AND A. KISTNER \\ National Chemical Research Laboratory, South African Council for \\ Scientific and Industrial Research - Onderstepoort Joint Unit for \\ Research on Digestion and Metabolism in Ruminants, P.O. Box 395, \\ Pretoria, South Africa
}

(Received I9 December 1972; revised 2 May 1973)

\begin{abstract}
SUMMARY
The growth responses of 33 strains of cellulolytic butyrivibrios, isolated from the rumens of sheep fed on low-protein teff hay diets, to various combinations of volatile fatty acids (VFA) have been determined. Propionate alone was generally retardatory to the acetate-utilizing strains whilst acetate was stimulatory. Branched-chain VFA were generally stimulatory to those strains on which propionate had a retardatory effect, and retardatory to those strains on which propionate had no effect. Propionate alone was generally stimulatory to the acetate-producing strains and the effect of acetate and branched-chain VFA was variable. The major nutrient requirements of a few selected acetate-utilizing and acetate-producing strains are presented. A chemically defined medium was formulated which permits as good growth as a rumen-fluid medium for the acetateutilizing butyrivibrios, Ruminococcus albus and Cillobacterium cellulosolvens.
\end{abstract}

\section{INTRODUCTION}

The anaerobic, Gram-negative, butyric acid-producing, curved rods which occur in the rumen of cattle and sheep were described by Bryant \& Small (I956), who established the genus Butyrivibrio to accommodate this group of bacteria. Only a single species, Butyrivibrio fibrisolvens, was defined. These bacteria appear to be among the most common in the rumens of cattle and sheep fed widely different diets (Hungate, 1950; Bryant \& Small, 1956; Shane, Gouws \& Kistner, 1969). They have also been isolated from wild ruminants (Hungate, Philips, McGregor, Hungate \& Buechner, 1959). Despite the fact that only a small proportion of isolates obtained from non-selective media bring about a detectable solubilization of cellulose in vitro (Bryant \& Small, 1956; Hungate, 1966; Latham, Sharpe \& Sutton, I97I), they have been found to be among the predominant cellulose digesters in cattle and sheep on poor hay diets (Hungate, 1957; 1966; Shane et al. 1969; van Gylswyk, 1970).

The characteristics of the Butyrivibrio isolates so far studied in detail vary much, and the classification of the genus is not very satisfactory. The characteristics of 17 of the 48 isolates studied by Bryant \& Small (I956) differed not only from the type species, but so widely from each other that the authors found it impractical to define further species. Hungate (I966) proposed a second species, Butyrivibrio alactacidigens, for strains which do not produce lactic acid in the fermentation of carbohydrate. Shane et al. (I969) divided their Butyrivibrio isolates into two groups: group I, containing isolates which utilized acetate and produced high lactate and low formate levels in the fermentation of carbohydrate; 
and group 2, containing isolates which produced acetate with low lactate and high formate levels. The group I isolates were closely related in their characteristics and differed only in minor respects from $B$. fibrisolvens (Bryant \& Small, 1956). None of the group I or 2 isolates could be identified with $B$. alactacidigens (Hungate, 1966).

Van Gylswyk (1970) showed that supplementation of low-protein teff hay with branchedchain volatile fatty acids (VFA) gave an increase in the numbers of cellulolytic ruminococci without a concomitant rise in the number of cellulolytic butyrivibrios. The results of earlier workers (Bryant \& Small, I956; Gill \& King, I958; Lee \& Moore, 1959) indicated that the latter have no requirement for branched-chain VFA. However, most of the work was on acetate-utilizing strains only and no information was available on the effect of branched-chain acids on growth rate. It was felt desirable therefore to investigate more fully the effects of VFA on the growth response of butyrivibrios. The straight-chain acids were included in this study in view of the findings of Bryant \& Robinson (1962) that acetate is generally important for the nutrition of many species of rumen bacteria.

A chemically defined medium which allows as good growth as a rumen-fluid medium is a prerequisite for such a study, since otherwise stimulatory effects which may operate in the rumen could well be missed. Previous workers showed that some strains of Butyrivibrio can be grown in semidefined or defined media (Bryant \& Small, I956; Gill \& King, 1958; Lee \& Moore, 1959; Bryant \& Robinson, 1962; 1963; Shane, 1966). However, most of these were acetate-utilizing strains which are less fastidious in their nutritional requirements than the acetate-producing butyrivibrios (Shane, 1966). Furthermore rumen fluid was stimulatory in all cases, indicating that the media were not optimal. Because of these findings it was decided to design a defined medium which would support as dense and as rapid a growth as a rumen-fluid medium.

\section{METHODS}

Bacteria. Thirty-three strains of cellulolytic butyrivibrios were used. All strains were isolated from the $10^{-6}$ to $10^{-8}$ dilutions of rumen contents of sheep fed on a diet consisting mainly of low-protein teff hay. Strain $\mathrm{s} 7$ was isolated in 1962 by L. Gouws (unpublished results). All the strains with code numbers ce and cxs were isolated in I963 (Shane, I966; Shane et al. 1969). Strains I I and I 2 were isolated in 1965 and the remaining strains in I966 (van Gylswyk, 1968, 1970).

Maintenance medium. Cellobiose-rumen fluid agar medium $(0.5 \%)$ was prepared as described by van Gylswyk \& Hoffman (1970).

Design of a chemically defined 'complete' medium. An attempt was made to formulate a chemically-defined medium to support the growth of all the cellulolytic species thus far isolated from sheep fed on teff hay. The qualitative composition of the medium was based on information abstracted from the literature. To eliminate growth inhibition or retardation due to imbalances, the concentrations and proportions of the components were based on their concentrations in rumen fluid, the extent of their utilization from media containing rumen fluid, or the chemical composition of rumen bacteria (Table I).

Culture media for general nutritional requirements. A basal medium (I) consisting of minerals, cellobiose, sodium bicarbonate, cysteine $\mathrm{HCl} \cdot \mathrm{H}_{2} \mathrm{O}$, dithiothreitol and indigo carmine was prepared using the concentrations given in Table I. Stock mixtures of vitamins, straight-chain VFA, branched-chain VFA, purines and pyrimidines, ammonium sulphate and amino acids were prepared and added to the basal medium to give all desired combinations of nutrients. Final concentrations were always as shown in Table I. Where rumen fluid was used, this was filtered (Millipore GS membrane) and added to give a final concentration 
Table I. Composition and rationale for the formulation of a chemically defined medium for cellulolytic bacteria

\author{
$\begin{array}{ccc}\mathrm{mg} / \mathrm{IOC} \mathrm{ml} & \text { medium }\end{array}$
}

$\left.\begin{array}{lr}\mathrm{KH}_{2} \mathrm{PO}_{4} ; \mathrm{K}_{2} \mathrm{HPO}_{4} ; \mathrm{NaCl} & 90 \cdot 0 \\ \mathrm{CaCl}_{2} ; \mathrm{MgCl}_{2} \cdot 6 \mathrm{H}_{2} \mathrm{O} & 2 \cdot 0 \\ \mathrm{MnCl}_{2} \cdot 4 \mathrm{H}_{2} \mathrm{O} & 1 \cdot 0 \\ \mathrm{FeSO}_{4} \cdot 7 \mathrm{H}_{2} \mathrm{O} & 0.4 \\ \mathrm{CuSO}_{4} \cdot 5 \mathrm{H}_{2} \mathrm{O} ; \mathrm{ZnSO}_{4} \cdot 7 \mathrm{H}_{2} \mathrm{O} & 0 . \mathrm{I} \\ \mathrm{CoCl}_{2} \cdot 6 \mathrm{H}_{2} \mathrm{O} & 0.0 \mathrm{I}\end{array}\right\} \quad$ Based on Bryant \& Robinson (1962)

500

$\begin{array}{ll}\mathrm{NaHCO}_{3} & 800 \\ \left(\mathrm{NH}_{4}\right)_{2} \mathrm{SO}_{4} & 150\end{array}$

Valine

Leucine

Isoleucine

Serine

Glycine

Methionine

Alanine

Threonine

Aspartic acid

Lysine

Phenyl alanine

Tyrosine

Histidine

Glutamic acid

Arginine

Proline

Tryptophan

Adenine

Guanine

Cytosine

Uracil

Thymine

Acetic acid

Propionic acid

Iso-butyric acid

Iso-valeric acid

2-Methyl butyric acid

Thiamine- $\mathrm{HCl}$

Riboflavin

Ca-pantothenate

Nicotinic acid

Nicotinamide

Folic acid

Vitamin B 12

Biotin

Pyridoxal-HCl

Pyridoxamine-2HCl

Pyridoxine- $\mathrm{HCl}$

p-Amino benzoic acid

Thioctic acid

Menadione

Cysteine $\mathrm{HCl} . \mathrm{H}_{2} \mathrm{O}$

Dinthiothreitol

Indigo carmine

Gas phase
Substrate utilizable by all cellulolytic rumen bacteria

Gives a pH of 6.8 with $98 \% \mathrm{CO}_{2} / 2 \% \mathrm{H}_{2}$

Excess for synthesis of $12.5 \mathrm{mg}$ cell-N/100 ml medium
$4 \cdot 8 *$

$5 \cdot 6^{*}$

$4 \cdot 5^{*}$

$2 \cdot 6^{*}$

$3 \cdot 7^{*}$

$2 \cdot 1^{*}$

$5 \cdot 0^{*}$

$3 \cdot 9^{*}$

$8 \cdot 4^{*}$

$5 \cdot 5^{*}$

$3 \cdot 8^{*}$

$3 \cdot 7^{*}$

I. 4 *

$9 \cdot 7^{*}$

$3 \cdot 8 *$

2.8*

$\mathrm{I} \cdot 2+$

$2 \cdot 0+$

$2 \cdot 0$

$2 \cdot 0 \ddagger$

$1 \cdot 5 \ddagger$

0.5

I 6.9

$6.7\}$

$-3 \cdot 6$

4.3

3.5

0.001

0.020

0.060

0.100

$0.100 \$$

0.005

0.020

0.005

o.cio

0.010

0.010|

0.005 ?

0.005

0.020

50

4.0

0.5

$98 \% \mathrm{CO}_{2}$

Total non-ammonia nitrogen: $12.5 \mathrm{mg} / 100 \mathrm{ml}$ medium (based on synthesis by Ruminococcus albus in $40 \%$ rumen fluid $/ \mathrm{I} \%$ carbohydrate media (van Gylswyk \& Labuschagne, 1970)

Based on maximum uptake from medium (van Gylswyk \& Hoffman, 1970; van Gylswyk \& Roché, 1970)

Based on the concentrations of the branched-chain amino acids from which these are derived by deamination and decarboxylation

Vitamin concentrations based on the contents of rumen bacterial cells (Porter, 196I)

$2 \% \mathrm{H}_{2}$

* Proportions of the amino acids based on composition of hydrolysates of rumen microbes (mean of four diets) (Weller, 1957).

+ Tryptophan based on Block \& Mitchell (1946-1947).

$\ddagger$ Proportions of purines and pyrimidines based on composition of bacterial cells (Herbert, 196r). Nucleic acid $\mathrm{N}$ can account for $20 \%$ of the total $\mathrm{N}$.

$\S$ Concentration as for nicotinic acid (Porter, 196I).

II Concentration as for vitamin B6 (Porter, 1961).

I. Concentration as for folic acid (Porter, 1961). 


\section{C. ROCHÉ, H. ALBERTYN, N. O. VAN GYLSWYK AND A. KISTNER}

of $40 \%(\mathrm{v} / \mathrm{v})$. The resulting mixtures were then gassed with $98 \% \mathrm{CO}_{2} / 2 \% \mathrm{H}_{2}$, and distributed aseptically in $\mathrm{IO} \mathrm{ml}$ amounts into sterile, $\mathrm{CO}_{2}$-gassed $\mathrm{I}$ oz McCartney bottles.

Culture media for VFA requirements. A basal medium was prepared containing all the nutrients listed in Table I, except the VFA and branched-chain amino acids. Portions $(5 \mathrm{ml})$ of stock solutions of the sodium salts of acetic ( $30 \mathrm{mmole} / \mathrm{l})$, propionic (Io mmole/1), isobutyric, $n$-valeric, iso-valeric and 2-methyl butyric $(0.02 \mathrm{mmole} / 1 \mathrm{each})$ acids were mixed to give the desired combinations, and made up to 11 with basal medium. The resulting media were then gassed and distributed as described above.

Anaerobic techniques and preparation of washed cell suspensions. These were as described by Roché, Albertyn \& Kistner (I973). For each experiment a fresh inoculum was prepared. A cell suspension $(0 \cdot \mathrm{I} \mathrm{ml})$ was injected into Io $\mathrm{ml}$ of test medium.

Measurement of growth. The inoculated media were incubated at $38{ }^{\circ} \mathrm{C}$ and the extinctions were read directly on the $\mathrm{I} \mathrm{oz} \mathrm{McCartney} \mathrm{bottles} \mathrm{(previously} \mathrm{matched)} \mathrm{at} 578 \mathrm{~nm}$ at $24 \mathrm{~h}$ intervals. The light path length was approximately $24 \mathrm{~mm}$.

Microscopic examination. All positive tests were checked microscopically, using Gramstained smears, for the absence of contamination.

Expression of results. In assessing growth the maximum extinctions attained and the time taken to reach them were taken into account.

In describing the various responses of micro-organisms to nutrients, different authors have used certain terms with different meanings. We shall describe nutrients as 'stimulatory' when their addition reduces the time required by the culture to reach peak growth, without affecting the final population density, and as 'retardatory' where their addition prolongs this time.

\section{RESULTS}

\section{Growth response to major groups of nutrients}

To check whether the medium formulated in Table I allowed good growth of the cellulolytic butyrivibrios, a few selected group I and group 2 butyrivibrios were inoculated into the 'complete' defined medium (Table I) and also into a series of media from which various combinations of classes of nutrients had been omitted. Two non-defined, habitat-simulating media, which were assumed to meet all nutritional requirements of the test organisms, were included for comparison. Medium B consisted of the basal medium (I, Methods) plus $\left(\mathrm{NH}_{4}\right)_{2} \mathrm{SO}_{4}$ and $40 \%$ (v/v) rumen fluid, whilst medium A contained all the nutrients listed in Table I plus $40 \%(\mathrm{v} / \mathrm{v})$ rumen fluid.

Tables showing all the media used and the complete set of results obtained are available on request from the authors; only the more significant observations are stated here.

Group I butyrivibrios. The four group I strains tested (I 2, 20, 24 and CE5I) grew well in medium B. The peak extinctions attained were between $\mathrm{I} \cdot \mathrm{O}$ and $\mathrm{I} \cdot 5$ for strain 20 and $>\mathrm{I} \cdot 5$ for the other strains, and were reached within $24 \mathrm{~h}$ of inoculation. Simultaneous addition of the remaining groups of nutrients (medium A) did not improve growth. The simplest of the defined media tested which supported growth of all four strains contained the basal medium plus vitamins and either $\left(\mathrm{NH}_{4}\right)_{2} \mathrm{SO}_{4}$ (medium $\mathrm{JN}$ ) or amino acids (medium JA). Growth on these media was poorer than on medium B; the maximum extinction was lower and was reached after a longer incubation period. When, however, both $\left(\mathrm{NH}_{4}\right)_{2} \mathrm{SO}_{4}$ and amino acids were added to the basal medium plus vitamins (medium JAN), growth was as good as on medium B. Contrary to expectations, addition of branched-chain VFA to medium $\mathrm{JN}$ did not improve growth but caused marked retardation of growth and, except in the case of strain CE5I, some inhibition. The retardatory and inhibitory action was also noted when 
branched-chain VFA were added to medium JA and, to a lesser extent, to medium JAN. Further addition of straight-chain VFA reversed the deleterious effect of the branchedchain VFA to a greater or lesser extent. For example, in three of the four strains addition of straight-chain as well as branched-chain VFA to medium JN gave better growth than on medium JN alone, and in one case growth was as good as on media JAN and B. However, strain 24 , which was very severely retarded and partially inhibited by the addition of branchedchain VFA, still grew poorer after the further addition of straight-chain acids than it did on medium JN alone. Addition of straight-chain acids alone to media JN and JA stimulated and/or increased the peak extinction in most cases, whereas supplementation of medium JAN with straight-chain VFA had no effect on growth.

The inhibitory effect of an addition of a single group of nutrients was also found with purines and pyrimidines which, when added to medium $\mathrm{JN}$, were retardatory and/or slightly inhibitory. Conversely, when added to medium JA they were generally stimulatory. They also partially reversed the retardatory effects of branched-chain VFA added to media $\mathrm{JN}$ and $\mathrm{JA}$, but were less effective than the straight-chain VFA.

No combination of purines and pyrimidines, straight-chain VFA and branched-chain VFA, added to media JN, JA or JAN, gave better growth than medium JAN alone. This medium is thus the best defined medium found in the present study for the growth of group I butyrivibrios.

Group 2 butyrivibrios. The three group 2 strains tested (CE49, CE60 and CE78) differed far more from one another than did the group I strains in their growth responses on the various media. They also grew to a lower density on the basal rumen-fluid/ $\left(\mathrm{NH}_{4}\right)_{2} \mathrm{SO}_{4}$ medium (medium B) than did the strains of group I, the maximum extinction being less than 0.5 for strain CE 49 and between $\mathrm{I} \cdot \mathrm{O}$ and $\mathrm{I} \cdot 5$ for strains CE6O and CE78. These densities were reached within $24 \mathrm{~h}$ of incubation. Like the group I isolates, they required only vitamins and either $\left(\mathrm{NH}_{4}\right)_{2} \mathrm{SO}_{4}$ or amino acids in addition to the basal mixture. Two of the strains grew as well on medium JAN as on medium B, but the third grew more slowly on the defined medium, reaching maximum density only after 5 days.

\section{Growth response to various combinations of VFA}

The basal medium for this part of the investigation contained all the nutrients listed in Table I, except straight-chain and branched-chain amino acids. The latter were omitted since they might mask a response to branched-chain VFA which would have been missed in the earlier part of the work where the complete mixture of amino acids was either included in, or omitted from, the medium. The growth response of the group I and group 2 butyrivibrio strains to the basal medium was compared with that to the basal medium plus propionate alone and propionate plus all possible combinations of acetate, iso-butyrate, 2methyl butyrate, iso-valerate and valerate. Each strain was tested at least twice on the 33 media. In most cases the duplicate runs gave identical results both with regard to the maximum growth attained and the time taken to reach this. Where this was not the case tests were repeated.

Group I butyrivibrios. As was the case with their general nutrient requirements, the group I butyrivibrios were remarkably homogeneous in their response to different combinations of VFA. The most striking finding was the unexpected response to propionate which alone was inhibitory to one strain (strain I8), retardatory to I9 strains and had no effect on the remaining seven strains tested. Acetate completely overcame the inhibitory or retardatory effects of propionate, and in fact gave more rapid growth than the basal medium alone. The effect of the branched-chain VFA and valerate, as a group, was not quite so clear-cut. 
For strain 18 they were retardatory. They were generally retardatory for those strains on which propionate had no effect and were generally stimulatory for those strains on which propionate had a retardatory effect. There were only slight differences between the strains when different combinations of the branched-chain VFA and valerate were provided.

Group 2 butyrivibrios. The group 2 butyrivibrios were a far more heterogeneous group than group I, and differed markedly from them in their response to the various media tested. They grew in the absence of VFA, but much more slowly than group I, and never attained the same peak extinctions. A major difference between them and group I was that propionate alone was generally stimulatory. The effect of acetate in media containing propionate, but none of the branched-chain VFA or valerate, was variable, ranging from complete inhibition in the case of strain CE66, to stimulation plus an increase in the final population density with strain II. None of the branched-chain VFA or valerate showed any marked effect, except for strains CE65 and CE66, where 2-methyl butyrate was essential in the presence of the other branched-chain acids and valerate. There were more interactions between combinations of branched-chain VFA than in the group I butyrivibrios, but no definite patterns emerged.

\section{Growth of other cellulolytic rumen organisms}

Since one of the aims of this work was to develop a chemically defined medium for the cultivation of as many species of cellulolytic rumen bacteria as possible, the complete medium (Table I) was also tested with other cellulolytic bacteria isolated in this laboratory. Ruminococcus albus and Cillobacterium cellulosolvens grew very well but $R$. flavefaciens and atypical ruminococci failed to grow. The cause of this failure is being investigated. Bacteroides succinogenes was not tested, but in view of the known nutritional requirements of this organism (Bryant \& Doetsch, 1955; Bryant, Robinson \& Chu, 1959), it should grow well on the medium described in Table I, with the addition of $n$-valeric acid.

\section{DISCUSSION}

The idea of developing a balanced, chemically defined medium to permit the growth of all cellulolytic rumen bacteria has been moderately successful, and it is now possible to grow most of the cellulolytic organisms, with the exception of Ruminococcus flavefaciens, on the same defined medium.

Our results show that the butyrivibrios have no requirement for VFA in general and the branched-chain VFA in particular. In fact the latter are frequently retardatory. This is in complete contrast to the ruminococci where one or more branched-chain VFA are essential (Bryant \& Doetsch, 1955; Allison, Bryant \& Doetsch, 1962). This lack of a requirement for the branched-chain acids provides an explanation for the predominance of Butyrivibrio in the rumen of sheep fed low-protein diets.

The stimulatory effect of acetate on the group I butyrivibrios suggests that glycolysis is the rate-limiting step for biosynthesis in the early stages of growth. This is supported by the finding of Kistner \& Kotze (I973) of very low activities of phosphofructokinase and pyruvate kinase in extracts of strain CE5I. Van Gylswyk (unpublished data) has also demonstrated pyruvate synthase activity in toluene-treated cells of strain I2. In the case of the group 2 strains, acetate was generally non-stimulatory. However, no enzyme studies have been carried out to explain this.

The retardatory effect of propionate on the group I butyrivibrios may be due to competition, in the early stages of growth, for CoA between the excess of propionate supplied and 
the slight amount of acetate initially present in the cell, as suggested by Hill (1952) and Pennington (1957). This of course should seldom occur in the rumen where exogenous acetate is normally far in excess of propionate.

The stimulatory effect of propionate on the group 2 butyrivibrios clearly indicates a marked difference in metabolic pathways between the two groups. This is also shown by end-product analyses. The group I butyrivibrios produced an average of 3 mmole butyrate/ 1 in media containing acetate and propionate only, and this fell to an average of $0.7 \mathrm{mmole} / \mathrm{l}$ when propionate alone or no VFA were provided. The group 2 butyrivibrios on the other hand produced only $\mathrm{I} \cdot 3$ mmole butyrate/l, whether or not acetate was present in the medium. This suggests that they cannot utilize exogenous acetate. The metabolic pathways in the group I and 2 butyrivibrios are at present under investigation in this laboratory.

An extremely good correlation was found between the division of the butyrivibrios into two groups on the basis of acetate utilization or production and their pattern of growth in the various media tested. For example, strain II, which had never been previously characterized, appeared to belong to group 2 on the basis of its growth response to the media used. Detailed analysis of its end-products showed that it did not utilize acetate and thus should be classified in group 2. This is in agreement with Shane et al. (I969), who felt that a better basis for dividing the genus Butyrivibrio into species than the scheme based on production or lack of production of lactic acid, was acetate utilization with 'high lactate' and 'low formate' or acetate production with 'low lactate' and 'high formate'.

The authors wish to thank Dr H. M. Schwartz for advice and interest in this work and Mr S. G. Kock for technical assistance.

\section{REFERENCES}

Allison, M. J., Bryant, M. P. \& Doetsch, R. N. (1962). Studies on the metabolic function of branchedchain volatile fatty acids, growth factors for ruminococci. I. Incorporation of isovalerate into leucine. Journal of Bacteriology 83, 523-532.

Block, R. J. \& Mitchell, H. H. (I946-47). The correlation of the amino-acid composition of proteins with their nutritive value. Nutrition Abstracts and Reviews 16, 249-278.

Bryant, M. P. \& Doetsch, R. N. (1955). Factors necessary for the growth of Bacteroides succinogenes in the volatile acid fraction of rumen fluid. Journal of Dairy Science 38, 340-350.

Bryant, M. P. \& Robinson, I. M. (1962). Some nutritional characteristics of predominant culturable ruminal bacteria. Journal of Bacteriology 84, 605-6I4.

Bryant, M. P. \& Robinson, I. M. (I963). Apparent incorporation of ammonia and amino-acid carbon during growth of selected species of ruminal bacteria. Journal of Dairy Science 46, I 50-1 54.

BRyant, M. P., Robinson, I. M. \& CHU, H. (1959). Observations on the nutrition of Bacteroides succinogenes - a ruminal cellulolytic bacterium. Journal of Dairy Science 42, I 83 I-1 847 .

Bryant, M. P. \& Small, N. (1956). The anaerobic monotrichous butyric acid-producing curved rod-shaped bacteria of the rumen. Journal of Bacteriology 72, 16-21.

Gill, J. W. \& KinG, K. W. (I958). Nutritional characteristics of a Butyrivibrio. Journal of Bacteriology $\mathbf{7 5}$, $666-673$.

Herbert, H. (196I). The chemical composition of micro-organisms as a function of their environment. Symposium of the Society for General Microbiology II, 39I-414.

Hill, C. H. (1952). Studies on the inhibition of growth of Streptococcus faecalis by sodium propionate. Journal of Biological Chemistry 199, 329-332.

Hungate, R. E. (1950). The anaerobic mesophilic cellulolytic bacteria. Bacteriological Reviews $\mathbf{1 4}$, I-50.

Hungate, R. E. (I957). Micro-organisms in the rumen of cattle fed a constant ration. Canadian Journal of Microbiology 3, 28, -3 I I.

Hungate, R. E. (I966). The Rumen and its Microbes, ist edn. New York and London: Academic Press.

Hungate, R. E., Philips, G. D., McGregor, A., Hungate, D. P. \& Buechner, H. K. (1959). Microbial fermentation in certain mammals. Science, New York 130, 1192-I I94. 
KISTNER, A. \& KoTzE, J. P. (I973). Enzymes of intermediary metabolism of Butyrivibrio fibrisolvens and Ruminococcus albus grown under glucose limitation. Canadian Journal of Microbiology (in the Press).

Latham, M. J., Sharpe, M. E. \& Sutton, J. D. (1971). The microbial flora of the rumen of cows fed hay and high cereal rations and its relationship to the rumen fermentation. Journal of Applied Bacteriology 34, 425-434.

LeE, H. C. \& MOORE, W. E. C. (1959). Isolation and fermentation characteristics of strains of Butyrivibrio from ruminal ingesta. Journal of Bacteriology 77, 741-747.

PenNington, R. J. (I957). Some effects of propionate upon acetate metabolism in animal tissue slices. Biochemical Journal 65, 534-540.

PORTER, J. W. G. (I96I). Vitamin synthesis in the rumen. In Digestive Physiology and Nutrition of the Ruminant, pp. 226-234. Edited by D. Lewis. London: Butterworth.

Roché, C., Albertyn, J. \& Kistner, A. (1973). A method of preparing washed suspensions of anaerobic bacteria for metabolic studies. Laboratory Practice (in the Press).

Shane, B. S. (1966). The Predominant Cellulolytic Rumen Bacteria of Sheep Fed Low-Protein Teff Hay. Ph.D. Thesis, University of Witwatersrand.

Shane, B. S., Gouws, L. \& Kistner, A. (1969). Cellulolytic bacteria occurring in the rumen of sheep conditioned to low-protein teff hay. Journal of General Microbiology 55, 445-457.

van GylswyK, N. O. (1968). The Cellulolytic Rumen Bacteria of Sheep Fed Supplemented Hay Diets. M.Sc. Thesis, University of Pretoria.

VAN GyLSWYK, N. O. (1970). The effect of supplementing a low-protein hay on the cellulolytic bacteria in the rumen of sheep and on the digestibility of cellulose and hemicellulose. Journal of Agricultural Science, Cambridge 74, 169-180.

van Gylswyk, N. O. \& Hoffman, J. P. L. (1970). Characteristics of cellulolytic cillobacteria from the rumens of sheep fed teff (Eragrostis tef) hay diets. Journal of General Microbiology 6o, 38I-386.

VAN GYLSWYK, N. O. \& LABUSChaGNE, J. P. L. (I97I). Relative efficiency of pure cultures of different species of cellulolytic rumen bacteria in solubilizing cellulose in vitro. Journal of General Microbiology 66, I09-II 3.

van Gylswyk, N. O. \& Roche, C. E. G. (1970). Characteristics of Ruminococcus and cellulolytic Butyribrio species from the rumens of sheep fed differently supplemented teff (Eragrostis tef) hay diets. Journal of General Microbiology 64, I I-I 7.

WELLER, R. A. (1957). The amino-acid composition of hydrolysates of microbial preparations from the rumen of sheep. Australian Journal of Biological Science 10, 384-389. 